\title{
Diffusional Kurtosis Imaging of the Corticospinal Tract in Multiple Sclerosis: Association with Neurologic Disability
}

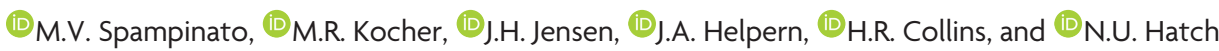

\begin{abstract}
BACKGROUND AND PURPOSE: Multiple sclerosis is an autoimmune disorder resulting in progressive neurologic disability. Our aim was to evaluate the associations between diffusional kurtosis imaging-derived metrics for the corticospinal tract and disability in multiple sclerosis.

MATERIALS AND METHODS: Forty patients with MS underwent brain MR imaging including diffusional kurtosis imaging. After we masked out T2 hyperintense lesions, the fractional anisotropy, mean diffusivity, radial diffusivity, axial diffusivity, mean kurtosis, radial kurtosis, and axial kurtosis were estimated for the corticospinal tract. Disability was quantified by using the Expanded Disability Status Scale at the time of MR imaging and 12 months post-MR imaging. The Pearson correlation coefficient and linear regression analyses were conducted to evaluate the associations between diffusion metrics and disability.

RESULTS: Significant correlations were found between the Expanded Disability Status Scale scores during the baseline visit and age $(r=$ 0.47), T2 lesion volume $(r=0.38)$, corticospinal tract mean diffusivity $(r=0.41)$, radial diffusivity $(r=0.41)$, axial diffusivity $(r=0.34)$, fractional anisotropy $(r=-0.36)$, and radial kurtosis $(r=-0.42)$. Significant correlations were also found between the Expanded Disability Status Scale scores at 12-month follow-up and age ( $r=0.38)$, mean diffusivity ( $r=0.45)$, radial diffusivity $(r=0.41)$, axial diffusivity ( $r=0.45)$, mean kurtosis ( $r=-0.42)$, radial kurtosis $(r=-0.56)$, and axial kurtosis $(r=-0.36)$. Linear regression analyses demonstrated significant associations among radial kurtosis, age, and Expanded Disability Status Scale score during the baseline visit, while radial kurtosis was the only variable associated with Expanded Disability Status Scale score for the 12-month follow-up.
\end{abstract}

CONCLUSIONS: Radial kurtosis of the corticospinal tract may have an association with neurologic disability in MS.

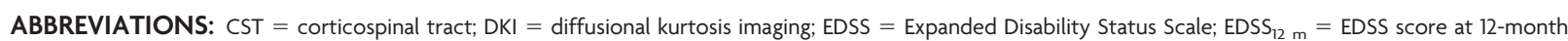
follow-up; $\mathrm{EDSS}_{0}=\mathrm{EDSS}$ at baseline visit; $\mathrm{FA}=$ fractional anisotropy; $\mathrm{K}_{\|}=$axial kurtosis; $\mathrm{K}_{\perp}=$ radial kurtosis; $\lambda_{\|}=$axial diffusivity; $\lambda_{\perp}=$ radial diffusivity; $\mathrm{MD}=$ mean diffusivity; MK = mean kurtosis; NAWM = normal-appearing white matter; $\mathrm{VIF}=$ variance inflation factor

M ultiple sclerosis is an autoimmune disorder of the central nervous system characterized by recurrent episodes of inflammation, demyelination, edema, and axonal loss, which can result in a progressive accumulation of neurologic disability. ${ }^{1} \mathrm{MR}$

Received December 28, 2016; accepted after revision March 14, 2017.

From the Department of Radiology and Radiological Science (M.V.S., M.R.K., J.H.J., J.A.H., H.R.C., N.U.H.) and Center for Biomedical Imaging (M.V.S., J.H.J., J.A.H.), Medical University of South Carolina, Charleston, South Carolina.

Paper previously presented in part at: Annual Meeting of the International Society for Magnetic Resonance in Medicine and European Society for Magnetic Resonance in Medicine, May 10-16, 2014; Milan, Italy; Annual Meeting of the American Society of Neuroradiology and the Foundation of the ASNR Symposium, May 1722, 2014; Montreal, Quebec, Canada; and Annual Meeting of the American Society of Neuroradiology and the Foundation of the ASNR Symposium, May-18-23, 2013; San Diego, California.

Please address correspondence to Maria Vittoria Spampinato, MD, Department of Radiology and Radiological Science, Medical University of South Carolina, 96 Jonathan Lucas, MSC 323, Charleston, SC 29425; e-mail: spampin@musc.edu; @mvspampinato

http://dx.doi.org/10.3174/ajnr.A5225 imaging is routinely performed to assess the burden of disease in patients with MS to guide therapeutic decisions. ${ }^{2}$ Conventional MR imaging is widely used to evaluate macrostructural changes such as T2 hyperintense lesions, T1 black holes, gadolinium-enhancing lesions, and brain volume loss. ${ }^{3,4}$ Although conventional MR imaging analysis has been used for years to assess the disease burden, it cannot fully explain the degree of neurologic dysfunction. The lack of correlation between neurologic disability and conventional MR imaging measures is commonly described as the "clinicoradiologic paradox."

DTI has been previously used to assess the integrity of brain tissue and to probe specific white matter tracts. ${ }^{5-12}$ Prior studies have evaluated microstructural changes of the corticospinal tract (CST) in MS and the association between diffusivity of the CST and measures of neurologic disability. ${ }^{5,7,13-17}$ However, a fundamental limitation of DTI is that the data analysis approximates the water diffusion dynamics within brain tissue as being a Gaussian 
process, though considerable non-Gaussian diffusion effects are observed throughout the brain. Hence, DTI does not fully characterize water diffusion in the brain. Diffusional kurtosis imaging $(\mathrm{DKI})^{18}$ is a clinically feasible diffusion MR imaging method, which extends the DTI model to include non-Gaussian diffusion effects. ${ }^{18-20}$ As a result, DKI has the potential to provide more sensitive biomarkers for probing microscopic structural changes occurring in the normal-appearing white matter (NAWM), which could lead to better predictive biomarkers for disease progression. Although DKI has been applied to MS in a few preliminary studies, ${ }^{18-22}$ the potential of DKI to aid in the assessment of neurologic disability in MS is still not well-established.

The aim of this study was to examine the associations between DKI-derived diffusion metrics of the corticospinal tract and physical disability in patients affected by MS, as measured by the Extended Disability Status Scale (EDSS). ${ }^{23}$ Our hypothesis was that DKI-derived metrics have stronger associations with neurologic dysfunction than DTI metrics.

\section{MATERIALS AND METHODS Participants}

The study was approved by the institutional review board, who exempted the study from requiring individual patient consent. We retrospectively reviewed the PACS for all patients with multiple sclerosis who underwent brain MR imaging during an 18month period. Inclusion criteria were the following: 1) age between 18 and 70 years; 2) diagnosis of multiple sclerosis according to established diagnostic criteria ${ }^{2}$; 3) brain MR imaging performed according to our standard multiple sclerosis protocol, including DKI; and 4) neurologic evaluation within 1 month of the MR imaging available in the electronic medical record (baseline visit). On the basis of the clinical phenotype, patients were categorized as having clinically isolated syndrome, relapsing-remitting MS, primary-progressive MS, secondary-progressive MS, or unknown type of MS. ${ }^{24}$ We excluded patients with history of neurologic disorders other than multiple sclerosis. We also excluded all imaging datasets degraded by bulk head motion or other artifacts. The degree of neurologic impairment was assessed by using the Kurtzke EDSS during the baseline visit $\left(\mathrm{EDSS}_{0}\right)$. If subsequent clinical neurologic encounters were documented in the electronic medical record, then we recorded the EDSS score for the neurologic visit 12 months after brain MR imaging (EDSS $12 \mathrm{~m}$ ).

\section{MR Imaging}

Brain MRIs were performed on a $1.5 \mathrm{~T}$ Avanto MR imaging scanner (Siemens, Erlangen, Germany). A sagittal T1-weighted MPRAGE $\left(\mathrm{TR} / \mathrm{TE} / \mathrm{TI}=1900 / 2.91 / 1100 \mathrm{~ms}\right.$, flip angle $=15^{\circ}$, acquisition matrix $=225 \times 199, \mathrm{FOV}=256 \times 256 \mathrm{~mm}^{2}$, section thickness $=1 \mathrm{~mm})$ and an axial FLAIR sequence $(\mathrm{TR} / \mathrm{TE} / \mathrm{TI}=$ $8000 / 88 / 2372 \mathrm{~ms}$, flip angle $=180^{\circ}$, matrix $=424 \times 512$, FOV $=$ $220 \times 220 \mathrm{~mm}^{2}$, section thickness $\left.=5 \mathrm{~mm}\right)$ were performed. Axial diffusion-weighted images were acquired with $3 \mathrm{~b}$-values $(0$, 1000 , and $2000 \mathrm{~s} / \mathrm{mm}^{2}$ ) along 30 diffusion-encoding directions with a single-shot twice-refocused spin-echo EPI sequence with $\mathrm{NEX}=1(\mathrm{NEX}=10$ for $b=0)$. Imaging parameters of the diffusion sequence were the following: voxel size $=3 \times 3 \times 3 \mathrm{~mm}^{3}$, number of sections $=40, \mathrm{TR} / \mathrm{TE}=5500 / 99 \mathrm{~ms}, \mathrm{FOV}=222 \times$
$222 \mathrm{~mm}^{2}$, acquisition matrix $=74 \times 74$, bandwidth/pixel $=1325$ $\mathrm{Hz}$, acceleration factor $=2$, acquisition time $\approx 7$ minutes.

\section{Data Processing}

The Lesion Segmentation Tool toolbox (http://www.appliedstatistics.de/lst.html), an open-source toolbox for SPM8 (http:// www.fil.ion.ucl.ac.uk/spm/software/spm12), specifically the lesion-growth algorithm, was used to segment T2 hyperintense lesions from the MPRAGE and FLAIR images $(\kappa$ value $=0.2){ }^{25}$ Then, a neuroradiologist blinded to all clinical information inspected each lesion mask to ensure accurate lesion segmentation. With MRIcron software (http://www.nitrc.org/projects/ mricron/), ${ }^{26}$ we calculated total white matter lesion volume for each patient.

With Diffusional Kurtosis Estimator software ${ }^{27}$ (Version 2.6, https://www.nitrc.org/projects/dke/) implemented in Matlab (MathWorks, Natick, Massachusetts), we calculated the diffusion and kurtosis tensors on a voxel-by-voxel basis. Parametric maps for the following metrics were obtained from the DKI dataset: 1) diffusivity metrics: fractional anisotropy (FA), mean diffusivity (MD), axial diffusivity $\left(\lambda_{\|}\right)$, and radial diffusivity $\left(\lambda_{\perp}\right)$ diffusivity; 2) kurtosis metrics: mean kurtosis $(\mathrm{MK})$, axial $\left(\mathrm{K}_{\|}\right)$and radial $\left(\mathrm{K}_{\perp}\right)$ kurtosis. MK is the average kurtosis over all diffusion directions, $K_{\|}$is the kurtosis in the direction of the diffusion tensor eigenvector with the largest diffusion eigenvalue (typically along the direction of the axons), and $\mathrm{K}_{\perp}$ is the average kurtosis over all directions perpendicular to the diffusion eigenvector with the largest eigenvalue (typically all directions perpendicular to the axons). ${ }^{18}$ FA maps were normalized to the Montreal Neurological Institute standard space FA template, a high-resolution average of 58 well-aligned good-quality FA images from healthy male and female subjects between 20 and 50 years of age, at $1 \times 1 \times 1 \mathrm{~mm}^{3}$ resolution. The resulting normalization parameters were then applied to the remaining diffusion maps and to the lesion masks. The white matter skeleton was obtained by using Tract-Based Spatial Statistics (TBSS; http://fsl.fmrib.ox.ac.uk/fsl/fslwiki/TBSS) in FSL (FA threshold, $\geq 0.4$ ). ${ }^{28}$ The corticospinal tract volumes of interest were obtained from the WM skeleton with the Johns Hopkins University White Matter Tractography Atlas, ${ }^{29}$ after masking out T2 hyperintense areas of individual patients (Fig 1). As a result, the CST VOI only included the NAWM of the CST. Then, average diffusion values of the CST VOIs were computed for all diffusion metrics and subjects by using Matlab.

Statistical analyses were conducted with SPSS (Version 22; IBM, Armonk, New York). Due to the small number of men in the study, sex differences in EDSS were examined with an independent-samples Mann-Whitney $U$ test. Pearson correlations were examined between CST diffusion metrics and EDSS at baseline and at the 12-month follow-up to characterize basic relations among variables. Results of the Pearson correlations were considered statistically significant with $P<.05$, corrected for multiple comparisons with the false discovery rate method. ${ }^{30}$ A linear regression equation with the stepwise method was calculated to evaluate the relationships among EDSS at baseline, the dependent variable, and the independent variables entered in the following order: age, white matter lesion volume, and CST diffusion param- 


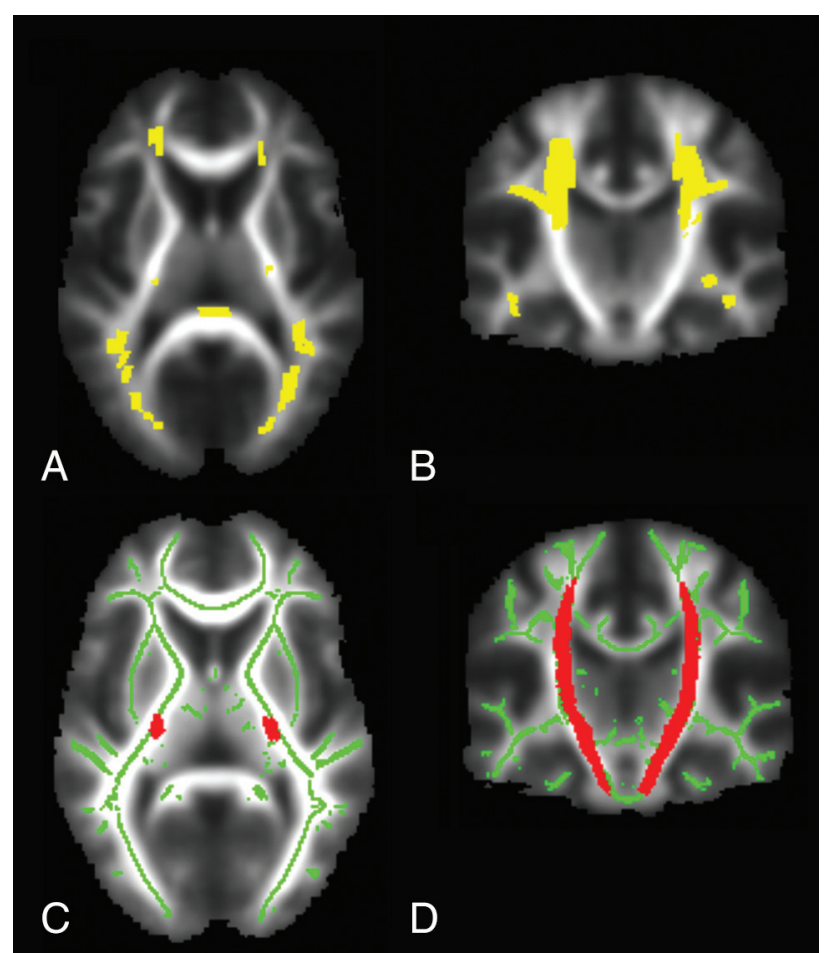

FIG 1. White matter lesion mask (yellow) of a representative patient with multiple sclerosis overlaid on axial $(A)$ and coronal $(B)$ mean fractional anisotropy images. The mean fractional anisotropy skeleton (green) and the Johns Hopkins University White Matter Tractography Atlas corticospinal tract (red) are overlaid onto the mean axial $(C)$ and coronal $(A)$ fractional anisotropy images. The white matter skeleton was obtained with FSL Tract-Based Spatial Statistics. The corticospinal tract VOIs were obtained from the white matter skeleton with the Johns Hopkins University White Matter Tractography Atlas, after masking out T2 hyperintense areas of individual patients. All images are in the Montreal Neurological Institute standard space.

eters. Additional linear regressions were also calculated to evaluate the associations between the same independent variables and EDSS at the 12-month follow-up and changes in the EDSS score between baseline and 12-month follow-up. Before the linear regression equation calculation, multicollinearity among independent variables was assessed with the variance inflation factor (VIF). Independent variables were excluded from the analysis when the VIF was $>10 .^{31}$ The results of the linear regression analyses were considered statistically significant at $P<.05$.

\section{RESULTS}

\section{Demographic and Clinical Characteristics}

Forty patients with MS were included in this study. Table 1 shows demographic, clinical, and conventional MR imaging characteristics of the entire cohort and of the subgroup of 28 patients with 12-month follow-up. There was no significant difference in EDSS scores between men and women at baseline (women: median $=3$, range $=1-8, n=33 ;$ men: median $=3$, range $=1-3.5, n=7 ; U=$ $138.5, P=.421$ ) and at the 12-month follow-up (women: median $=$ 3 , range $=0-9, n=24$; men: median $=6$, range $=1-6, n=4$; $U=39.5, P=.590)$. Patients had a disease duration of at least 1 year since their first symptom before the brain MR imaging. The exact duration of disease was not documented in the chart for 3 patients. Of the 40 patients, 25 were receiving disease-modifying
Table 1: Patient clinical characteristics and imaging metrics

\begin{tabular}{|c|c|c|}
\hline & $\begin{array}{l}\text { Entire } \\
\text { Cohort }\end{array}$ & $\begin{array}{l}\text { Patients with } \\
\text { 12-Month } \\
\text { Follow-Up }\end{array}$ \\
\hline No. of subjects & 40 & 28 \\
\hline $\operatorname{Sex}(F / M)$ & $33 / 7$ & $24 / 4$ \\
\hline Age (mean) (yr) & $42.9 \pm 12.5$ & $42.1 \pm 13.1$ \\
\hline $\mathrm{EDSS}_{0}$ (mean) & $3.4 \pm 1.9$ & $3.6 \pm 2$ \\
\hline $\mathrm{EDSS}_{12 \mathrm{~m}}$ (mean) & & $4.2 \pm 2.3$ \\
\hline MS type (RR/SP/unknown/PP) & $32 / 2 / 5 / 1$ & $23 / 1 / 3 / 1$ \\
\hline Disease duration (mean) (yr) & $8.0 \pm 6.4$ & $6.9 \pm 4.5$ \\
\hline $\mathrm{T} 2$ lesion load (mean) (mL) & $21.3 \pm 22.8$ & $23.3 \pm 26.0$ \\
\hline CST FA (mean) & $0.44 \pm 0.03$ & $0.44 \pm 0.03$ \\
\hline CST MD (mean) & $0.94 \pm 0.06$ & $0.93 \pm 0.06$ \\
\hline $\operatorname{CST} \lambda_{\perp}$ (mean) & $0.70 \pm 0.06$ & $0.70 \pm 0.06$ \\
\hline CST (mean) & $1.41 \pm 0.07$ & $1.40 \pm 0.07$ \\
\hline CST MK (mean) & $1.17 \pm 0.06$ & $1.17 \pm 0.06$ \\
\hline $\mathrm{CST} \mathrm{K}_{\perp}$ (mean) & $1.60 \pm 0.15$ & $1.60 \pm 0.14$ \\
\hline $\mathrm{CST} \mathrm{K}_{\|}($mean) & $0.96 \pm 0.05$ & $0.97 \pm 0.05$ \\
\hline
\end{tabular}

Note:- - RR indicates relapsing-remitting MS; SP, secondary-progressive MS; PP, primary-progressive MS.

Table 2: Correlations between EDSS and age, T2 lesion volume, and diffusion metrics

\begin{tabular}{|c|c|c|c|c|}
\hline \multirow{2}{*}{$\begin{array}{l}\text { Diffusion } \\
\text { Metrics }\end{array}$} & \multicolumn{2}{|c|}{$\mathrm{EDSS}_{0}$} & \multicolumn{2}{|c|}{$\mathrm{EDSS}_{12 \mathrm{~m}}$} \\
\hline & $r$ & $P$ Value ${ }^{a}$ & $r$ & $P$ Value $^{\mathrm{a}}$ \\
\hline Age & 0.47 & .009 & 0.38 & .003 \\
\hline T2 lesion volume & 0.38 & .018 & 0.23 & .117 \\
\hline$M D$ & 0.41 & .018 & 0.45 & .018 \\
\hline$\lambda_{\|}$ & 0.34 & .023 & 0.45 & .018 \\
\hline$\lambda_{\perp}^{\| \prime}$ & 0.41 & .018 & 0.41 & .023 \\
\hline FA & -0.36 & .022 & -0.30 & .063 \\
\hline MK & -0.29 & .046 & -0.42 & .023 \\
\hline $\mathrm{K}_{\|}$ & -0.25 & .063 & -0.36 & .040 \\
\hline $\mathrm{K}_{\perp}$ & -0.42 & .018 & -0.56 & .009 \\
\hline
\end{tabular}

${ }^{a} P$ values were corrected for multiple comparisons with the false discovery rate method.

treatments, which included interferon $\beta(n=13)$, glatiramer acetate $(n=8)$, natalizumab $(n=3)$, and fingolimod $(n=1)$.

\section{Imaging Data}

Table 2 demonstrates the correlations among age, T2 lesion volume, CST diffusion metrics, $\mathrm{EDSS}_{0}$, and $\mathrm{EDSS}_{12 \mathrm{~m}}$ (Fig 2). Analyses of the multicollinearity among independent variables resulted in inclusion in the regression model of the following independent variables with a VIF of $<10$ : age $(\mathrm{VIF}=1.30)$, T2 white matter lesion volume (VIF $=1.42)$, CST MK $(\mathrm{VIF}=$ $5.89)$, CST $K_{\|}(\mathrm{VIF}=2.21)$, and $\mathrm{CST} \mathrm{K}_{\perp}(\mathrm{VIF}=5.43)$. FA, $\mathrm{MD}, \lambda_{\perp}$, and $\lambda_{\|}$were excluded from the analyses due to a VIF of $>10$. Then, a linear regression analysis was performed to evaluate the relationship between $\mathrm{EDSS}_{0}$ and the following variables: age, $\mathrm{T} 2$ white matter lesion volume, $\mathrm{MK}, \mathrm{K}_{\|}$, and $\mathrm{K}_{\perp}$. A significant regression equation was found $(F[2,37]=8.875$, $P=.001)$ with an $R^{2}$ of $0.324 . \mathrm{K}_{\perp}(\beta=-0.329, P=.023)$ and age $(\beta=0.399, P=.007)$ were the only variables significantly associated with $\operatorname{EDSS}_{0}$ (Table 3 ). A linear regression analysis was performed to evaluate the relationship between EDSS $_{12 \mathrm{~m}}$ and the same variables (age, T2 lesion load, and CST diffusion metrics obtained at baseline). A significant regression equation was found $(F[1,26]=12.014, P=.002)$ with an $R^{2}$ of 0.316 . $\mathrm{K}_{\perp}(\beta=-0.562, P=.002)$ was the only variable to have a significant association with EDSS $_{12 \mathrm{~m}}$ (Table 3). A linear re- 

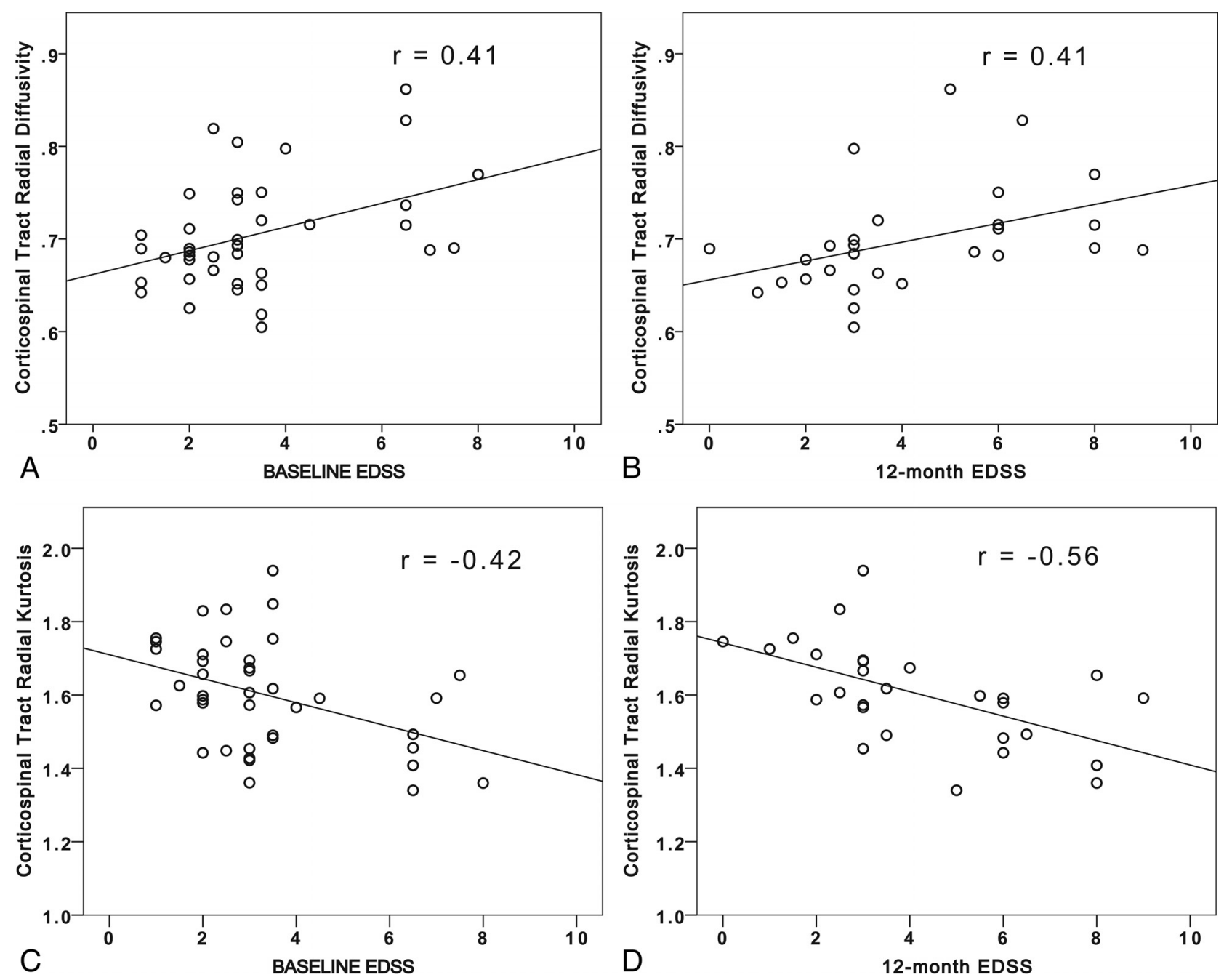

FIG 2. Scatterplots for the Expanded Disability Status Scale at the time of the brain MR imaging versus radial diffusivity $(A)$ and radial kurtosis (C) of the corticospinal tracts, and EDSS at the follow-up visit 12 months after the brain MR imaging versus radial diffusivity (B) and radial kurtosis (D) of the corticospinal tracts in patients with MS.

Table 3: Linear regression analyses: coefficients table

\begin{tabular}{lccccc}
\hline & \multicolumn{2}{c}{ EDSS $_{0}$} & & \multicolumn{2}{c}{ EDSS $_{12 \mathrm{~m}}$} \\
\cline { 2 - 3 } \cline { 5 - 6 } & $\boldsymbol{\beta}$ & $\boldsymbol{P}$ Value & & $\boldsymbol{\beta}$ & $\boldsymbol{P}$ Value \\
\hline (Constant) & & .022 & & $<.001$ \\
Age & .399 & .007 & & .172 & .347 \\
CST MK $_{\text {CST K }}$ & .074 & .811 & & .257 & .432 \\
CST K $_{\perp}$ & .055 & .774 & & .006 & .979 \\
T2 lesion volume &. .329 & .023 & & -.562 & .002 \\
\hline
\end{tabular}

gression analysis was also performed to evaluate the relationship between change in the EDSS score (baseline EDSS versus 12-month follow-up EDSS) and the same independent variables. None of the variables had significant associations with change in the EDSS score.

\section{DISCUSSION}

We conducted a retrospective study designed to explore the hypothesis that DKI-derived metrics of the CST have stronger associations with physical disability than DTI-derived metrics in patients with MS. We have preliminarily found that $\mathrm{K}_{\perp}$ of the CST has an association with physical disability. Associations were found between DKI-derived metrics and neurologic disability at the time of brain MR imaging, as well as with the degree of disability at 12-month follow-up. Due to the retrospective nature of our study, however, our results will require validation with future prospective studies. If our preliminary observations are confirmed, DKI-derived metrics may play an important role in improving our understanding of physical disability and prognosis, as well as in guiding treatment considerations in multiple sclerosis.

The correlations between DTI-derived measures of CST integrity and functional disability in MS have been previously

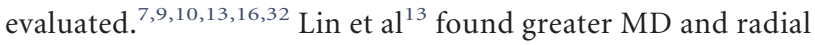
diffusivity in the normal-appearing CST in patients with MS than in healthy controls. Prior studies found significant associations between DTI-derived measures of CST integrity and functional disability. Bergsland et $\mathrm{al}^{32}$ found significant correlations between the EDSS and diffusivity measures, including FA, MD, $\lambda_{\perp}$, and $\lambda_{\|}$for the NAWM CST. Tovar-Moll et al ${ }^{5}$ found an inverse association between CST FA and EDSS when correcting for lesion volume. Other authors found only trends for an inverse correlation between EDSS and CST FA in 
$\mathrm{MS}^{14,15}$ or found weak correlations between EDSS and DTI metrics of the CST $\left(\mathrm{MD}, \lambda_{\perp}\right.$, and $\left.\lambda_{\|}\right) .{ }^{9}$ Reich et $\mathrm{al}^{16}$ did not find significant differences in FA, MD, $\lambda_{\perp}$, and $\lambda_{\|}$for the CST among patients with various degrees of ankle dorsiflexion impairment. Harrison et $\mathrm{al}^{33}$ found that DTI indices of the CST were not able to predict EDSS scores. Overall, prior study results demonstrated that conventional DTI measures may have an association with physical disability, but this relationship has not been consistently demonstrated in the literature. Here, we report associations of conventional DTI metrics for the CST with EDSS, which confirm prior studies, and extend prior investigations by demonstrating significant correlations between kurtosis metrics, especially $\mathrm{K}_{\perp}$, and EDSS.

The diffusional kurtosis metrics are complementary to the diffusion tensor metrics and provide microstructural information that more comprehensively characterizes brain tissue microstructure. ${ }^{18} \mathrm{~A}$ few studies have previously evaluated the use of DKIderived metrics in the characterization of demyelinating disease in animal models and in patients affected by MS. Using the cuprizone mouse model of CNS demyelination, Falangola et $\mathrm{al}^{34}$ evaluated the associations between DKI-derived metrics in cuprizone-induced chronic demyelination of the corpus callosum and morphologic WM alterations associated with myelin pathology. DKI-derived metrics were found to have greater sensitivity than conventional DTI parameters in the detection of cuprizone-induced demyelination of the anterior corpus callosum. Guglielmetti et $\mathrm{al}^{35}$ found that $\mathrm{MK}$ and $\mathrm{K}_{\perp}$, but not DTI-derived parameters, were sensitive to cuprizone-induced cortical alterations. Yoshida et $\mathrm{al}^{19}$ have conducted ROI-based quantitative analyses of MK for 24 cerebral NAWM regions in 11 patients with MS. The average MK for the NAWM was found to be lower in patients with MS than in healthy controls. Raz et $\mathrm{al}^{21}$ evaluated the signal characteristics of the spinal cord by using DKI and found that MK was lower in patients with MS than in healthy controls. Abnormal DKI-derived metrics of the NAWM were found in patients with MS but not in individuals with neuromyelitis optica. ${ }^{22}$ Bester et $\mathrm{al}^{20}$ applied DKI to the evaluation of cortical gray matter microstructural changes and found that decreased cortical MK was associated with poor performance on the Delis-Kaplan Executive Function System test $(r=0.66)$. De Kouchkovsky et al ${ }^{17}$ assessed white matter integrity in MS by using DKI-derived white matter integrity metrics, specifically axonal water fraction, intra-axonal diffusivity, radial and axial extra-axonal diffusivity, and tortuosity of the extra-axonal space. The authors found a moderate association between axonal water fraction in the corpus callosum and the EDSS score $(\rho=-0.39)$. Our study design differs from the methodology used by de Kouchkovsky et al. In fact, our approach was to study the associations between physical disability and pure diffusion metrics of the CST, such as mean kurtosis and radial kurtosis, as opposed to model-dependent quantities such as axonal water fraction.

These prior studies have shown that kurtosis parameters provide more comprehensive information about brain tissue microstructure than that available with conventional DTI. ${ }^{18}$ It is important to determine whether this enhanced sensitivity to microstructural changes provided by DKI translates into improved disability prediction. Our work demonstrates promising relations between DKI-derived diffusion metrics for the CST and clinical disability. In this retrospective cohort, age and $\mathrm{K}_{\perp}$ were associated with neurologic disability and accounted for approximately $32 \%$ of the variability of EDSS scores at baseline. We also found that $\mathrm{K}_{\perp}$ was the only parameter significantly associated with disability at the 12-month follow-up examination and accounted for about $32 \%$ of the variability of EDSS scores. If the associations between $\mathrm{K}_{\perp}$ and disability are validated in future larger prospective studies, these metrics may ultimately prove to be powerful adjuncts to the clinical assessment of patients with MS.

Histopathology and MR imaging studies have shown that the NAWM is affected, to a lesser extent, by the same pathologic processes that are found in MS lesions, specifically inflammation, demyelination, microglial activation, axonal injury, Wallerian degeneration, and macrophage infiltration. $^{36-39}$ The pathologic basis of the stronger association between $\mathrm{K}_{\perp}$ and physical disability in MS requires further determination. $K_{\perp}$ is the average kurtosis over all directions perpendicular to the diffusion eigenvector with the largest eigenvalue (typically all directions perpendicular to the axons). We hypothesized that the stronger association between physical disability and $\mathrm{K}_{\perp}$ than between other diffusion metrics may reflect a greater sensitivity of $\mathrm{K}_{\perp}$ to early pathologic changes of the NAWM, possibly myelin breakdown resulting in demyelination or hypomyelination or an increase of membrane barriers due to microgliosis. ${ }^{34,35}$ Unfortunately, pathologic findings for the NAWM are not available for this patient cohort. Future studies on the correlations between DKI parameters and pathologic findings for the NAWM in MS will help clarify the histopathologic basis of our preliminary observations.

An alternative approach to DKI for quantifying diffusional non-Gaussianity is to fit the diffusion MR imaging signal to a stretched exponential model. ${ }^{40}$ However, this model does not allow the estimation of kurtosis, ${ }^{18}$ and it is less appropriate for white matter because incorporating the effects of diffusion anisotropy is challenging. ${ }^{41}$ Biexponential fitting of the diffusion MR imaging signal is also feasible. This is fully consistent with DKI but requires the acquisition of more diffusion-weighted data than the truncated cumulant expansion signal model conventionally used with DKI (as in the present study). ${ }^{18}$

This study has limitations. First, it was a retrospective study that evaluated a relatively limited number of 40 patients, which precluded analysis of data in patients with relapsing-remitting, primary-progressive, and secondary-progressive MS separately. Twelve-month clinical follow-up information was available for only $28 / 40$ patients (70\%). We also did not evaluate the subsequent temporal evolution of the observed CST diffusion measures. Finally, while the EDSS is an effective tool widely used in clinical trials to assess the severity of MS, it is heavily weighted toward ambulation, and the multiple sclerosis functional composite may be a more sensitive method to evaluate disease progression. ${ }^{28}$

\section{CONCLUSIONS}

We have found significant associations between the $\mathrm{K}_{\perp}$ of the CST and disability in MS. DKI metrics were found to have stronger 
associations than DTI metrics with measures of disability in MS. It is important that this work be validated in future prospective studies with larger sample sizes.

\section{ACKNOWLEDGMENTS}

The authors gratefully acknowledge Ali Tabesh for his contributions to study design, image postprocessing, and data analysis and Dr. Aljoeson Walker for performing the neurologic examinations.

Disclosures: Jens H. Jensen-OTHER RELATIONSHIPS: I am a coinventor on US Patent 8811706 that covers one of the imaging methods investigated in this article (DKI). The patent is owned by a former employer (New York University), but I could be entitled to a share of any royalties at some point. To date, I have not received royalties from this patent. Joseph A. Helpern—UNRELATED: Grant: The Litwin Foundation*. Neal U. Hatch-UNRELATED: Support for Travel to Meetings for the Study or Other Purposes: partial reimbursement of travel expenses provided by the department. *Money paid to the institution.

\section{REFERENCES}

1. Compston A, Coles A. Multiple sclerosis. Lancet 2002;359:1221-31 CrossRef Medline

2. Polman CH, Reingold SC, Banwell B, et al. Diagnostic criteria for multiple sclerosis: 2010 revisions to the McDonald criteria. Ann Neurol 2011;69:292-302 CrossRef Medline

3. Filippi M, Rocca MA, Barkhof F, et al; Attendees of the Correlation between Pathological MRI findings in MS workshop. Association between pathological and MRI findings in multiple sclerosis. Lancet Neurol 2012;11:349-60 CrossRef Medline

4. Barkhof F. MRI in multiple sclerosis: correlation with Expanded Disability Status Scale (EDSS). Mult Scler 1999;5:283-86 Medline

5. Tovar-Moll F, Evangelou IE, Chiu AW, et al. Diffuse and focal corticospinal tract disease and its impact on patient disability in multiple sclerosis. J Neuroimaging 2015;25:200-06 CrossRef Medline

6. Sbardella E, Petsas N, Tona F, et al. Assessing the correlation between grey and white matter damage with motor and cognitive impairment in multiple sclerosis patients. PLoS One 2013;8:e63250 CrossRef Medline

7. Wilson M, Tench CR, Morgan PS, et al. Pyramidal tract mapping by diffusion tensor magnetic resonance imaging in multiple sclerosis: improving correlations with disability. J Neurol Neurosurg Psychiatry 2003;74:203-07 CrossRef Medline

8. Onu M, Roceanu A, Sboto-Frankenstein U, et al. Diffusion abnormality maps in demyelinating disease: correlations with clinical scores. Eur J Radiol 2012;81:e386-391 CrossRef Medline

9. Daams M, Steenwijk MD, Wattjes MP, et al. Unraveling the neuroimaging predictors for motor dysfunction in long-standing multiple sclerosis. Neurology 2015;85:248-55 CrossRef Medline

10. Inglese $M$, Bester $M$. Diffusion imaging in multiple sclerosis: research and clinical implications. NMR Biomed 2010;23:865-72 CrossRef Medline

11. Koenig KA, Sakaie KE, Lowe MJ, et al. The relationship between cognitive function and high-resolution diffusion tensor MRI of the cingulum bundle in multiple sclerosis. Mult Scler 2015;21:1794-801 CrossRef Medline

12. Preziosa P, Rocca MA, Mesaros S, et al. Relationship between damage to the cerebellar peduncles and clinical disability in multiple sclerosis. Radiology 2014;271:822-30 CrossRef Medline

13. Lin F, Yu C, Jiang T, et al. Diffusion tensor tractography-based group mapping of the pyramidal tract in relapsing-remitting multiple sclerosis patients. AJNR Am J Neuroradiol 2007;28:278-82 Medline

14. Giorgio A, Palace J, Johansen-Berg H, et al. Relationships of brain white matter microstructure with clinical and MR measures in relapsing-remitting multiple sclerosis. J Magn Reson Imaging 2010;31: 309-16 CrossRef Medline

15. Gorgoraptis N, Wheeler-Kingshott CA, Jenkins TM, et al. Combin- ing tractography and cortical measures to test system-specific hypotheses in multiple sclerosis. Mult Scler 2010;16:555-65 CrossRef Medline

16. Reich DS, Zackowski KM, Gordon-Lipkin EM, et al. Corticospinal tract abnormalities are associated with weakness in multiple sclerosis. AJNR Am J Neuroradiol 2008;29:333-39 CrossRef Medline

17. de Kouchkovsky I, Fieremans E, Fleysher L, et al. Quantification of normal-appearing white matter tract integrity in multiple sclerosis: a diffusion kurtosis imaging study. J Neurol 2016;263:1146-55 CrossRef Medline

18. Jensen JH, Helpern JA. MRI quantification of non-Gaussian water diffusion by kurtosis analysis. NMR Biomed 2010;23:698-710 CrossRef Medline

19. Yoshida M, Hori M, Yokoyama K, et al. Diffusional kurtosis imaging of normal-appearing white matter in multiple sclerosis: preliminary clinical experience. Jpn J Radiol 2013;31:50-55 CrossRef Medline

20. Bester M, Jensen JH, Babb JS, et al. Non-Gaussian diffusion MRI of gray matter is associated with cognitive impairment in multiple sclerosis. Mult Scler 2015;21:935-44 CrossRef Medline

21. Raz E, Bester M, Sigmund EE, et al. A better characterization of spinal cord damage in multiple sclerosis: a diffusional kurtosis imaging study. AJNR Am J Neuroradiol 2013;34:1846-52 CrossRef Medline

22. Qian W, Chan KH, Hui ES, et al. Application of diffusional kurtosis imaging to detect occult brain damage in multiple sclerosis and neuromyelitis optica. NMR Biomed 2016;29:1536-45 CrossRef Medline

23. Kurtzke JF. Rating neurologic impairment in multiple sclerosis: an expanded disability status scale (EDSS). Neurology 1983;33:1444-52 CrossRef Medline

24. Lublin FD, Reingold SC, Cohen JA, et al. Defining the clinical course of multiple sclerosis: the 2013 revisions. Neurology 2014;83:278-86 CrossRef Medline

25. Schmidt P, Gaser C, Arsic M, et al. An automated tool for detection of FLAIR-hyperintense white-matter lesions in multiple sclerosis. Neuroimage 2012;59:3774-83 CrossRef Medline

26. Rorden C, Karnath HO, Bonilha L. Improving lesion-symptom mapping. J Cogn Neurosci 2007;19:1081-88 CrossRef Medline

27. Tabesh A, Jensen JH, Ardekani BA, et al. Estimation of tensors and tensor-derived measures in diffusional kurtosis imaging. Magn Reson Med 2011;65:823-36 CrossRef Medline

28. Smith SM, Jenkinson M, Woolrich MW, et al. Advances in functional and structural MR image analysis and implementation as FSL. Neuroimage 2004;23(suppl 1):S208-19 CrossRef Medline

29. Hua K, Zhang J, Wakana S, et al. Tract probability maps in stereotaxic spaces: analyses of white matter anatomy and tract-specific quantification. Neuroimage 2008;39:336-47 CrossRef Medline

30. Benjamini Y, Hochberg Y. Controlling the false discovery rate: a practical and powerful approach to multiple testing. Journal of the Royal Statistical Society Series B (Methodological) 1995;57:289-300

31. Neter J, Wasserman W, Kutner MH. Applied Linear Regression Models. Homewood: R.D. Irwin; 1983

32. Bergsland N, Laganà MM, Tavazzi E, et al. Corticospinal tract integrity is related to primary motor cortex thinning in relapsing-remitting multiple sclerosis. Mult Scler 2015;21:1771-80 CrossRef Medline

33. Harrison DM, Shiee N, Bazin PL, et al. Tract-specific quantitative MRI better correlates with disability than conventional MRI in multiple sclerosis. J Neurol 2013;260:397-406 CrossRef Medline

34. Falangola MF, Guilfoyle DN, Tabesh A, et al. Histological correlation of diffusional kurtosis and white matter modeling metrics in cuprizone-induced corpus callosum demyelination. NMR Biomed 2014; 27:948-57 CrossRef Medline

35. Guglielmetti C, Veraart J, Roelant E, et al. Diffusion kurtosis imaging probes cortical alterations and white matter pathology following cuprizone induced demyelination and spontaneous remyelination. Neuroimage 2016;125:363-77 CrossRef Medline 
36. Evangelou N, Konz D, Esiri MM, et al. Regional axonal loss in the corpus callosum correlates with cerebral white matter lesion volume and distribution in multiple sclerosis. Brain 2000;123(pt 9): 1845-49 CrossRef Medline

37. Allen IV, McQuaid S, Mirakhur M, et al. Pathological abnormalities in the normal-appearing white matter in multiple sclerosis. Neurol Sci 2001;22:141-44 CrossRef Medline

38. Trapp BD, Peterson J, Ransohoff RM, et al. Axonal transection in the lesions of multiple sclerosis. $N$ Engl J Med 1998;338:278-85 CrossRef Medline
39. Evangelou N, Esiri MM, Smith S, et al. Quantitative pathological evidence for axonal loss in normal appearing white matter in multiple sclerosis. Ann Neurol 2000;47:391-95 Medline

40. Bennett KM, Schmainda KM, Bennett RT, et al. Characterization of continuously distributed cortical water diffusion rates with a stretched-exponential model. Magn Reson Med 2003;50:727-34 CrossRef Medline

41. Meerschaert MM, Magin RL, Ye AQ. Anisotropic fractional diffusion tensor imaging. J Vib Control 2016;22:2211-21 CrossRef Medline 